\title{
Influence of Spin-Orbit Interaction on Band Structure and Elastic Properties of PbTe
}

\author{
T. RADZYŃSKI AND A. ŁUSAKOWSKI \\ Institute of Physics, Polish Academy of Sciences, al. Lotników 32/46, 02-668 Warsaw, Poland

\begin{abstract}
Inclusion of spin-orbit interaction in density functional theory calculations of $\mathrm{PbTe}$ band structure leads to wrong results concerning the symmetry of valence and conduction band wave functions. Using two ab initio codes: OpenMX and Quantum Espresso we show that changing the spin-orbit coupling strength in pseudopotentials for $\mathrm{Pb}$ it is possible to obtain proper energy gap and wave functions of proper symmetries.
\end{abstract}

PACS numbers: 31.15.A-, 31.15.aj, 71.20.-b, 87.19.rd

\section{Introduction}

Lead telluride is very promising for spintronics application. It is characterized by a number of excellent semiconductor properties, such as a high carrier mobility or the possibility to significantly change the free carrier concentration. It can also be used as a basis to produce mixed crystals with other compounds with very interesting properties. For example, it is known that PbGeTe is ferroelectric and becomes also magnetic when additionally doped with manganese or europium. A coexistence of ferroelectricity and magnetism can be used, for example, to construct four-states memories. In order to calculate physical properties of these more complicated compounds it is first necessary to fully understand electronic properties of the basic compound, i.e., $\mathrm{PbTe}$, and to prepare tools to carry out efficient calculations in more complicated cases.

The direct energy gap for PbTe equals to $0.19 \mathrm{eV}$ and it is located in the $L$ point of the Brillouin zone. It is known that in the vicinity of the $L$ point the wave functions for the valence and conduction bands consist mainly of tellurium $5 p$ and lead $6 p$ orbitals, respectively. We calculated $\mathrm{PbTe}$ band structure applying density functional theory (DFT) methods. Using pseudopotentials generated by us (see below) and those distributed with $a b$ initio packages we obtained approximately correct values of the energy gap; however, the electronic structure was inverted which means that the valence band consisted mainly of lead $6 p$ orbitals. Such results are connected with the energy gap problem, frequently encountered in DFT calculations - the calculated energy gap is smaller than the experimentally determined value. If the spinorbit coupling was neglected in our calculations, we obtained $E_{\mathrm{g}} \approx 0.5 \mathrm{eV}$ and the proper band structure. However, the spin-orbit splitting of lead $6 p$ levels is so large that when it is turned on the energy of $6 p$ lead orbitals lie below energy of $5 p$ tellurium orbitals. This mechanism leads to the inverted band structure - the lead $6 p$ orbitals become valence orbitals.

The main reason of the energy gap problem lies in the approximated Kohn-Sham potential used in DFT calculations. In order to overcome this problem one should use better approximation to the exchange-correlation potential like for example hybrid functional [1] or exact exchange formalism [2]. However the codes we use do not offer such possibilities. Another approach was proposed in Ref. [3] where the authors corrected the energy gap error applying a constant potential to the conduction band states. In the present work we show that if we properly change the strength of spin-orbit interaction we obtain the energy band structure for $\mathrm{PbTe}$ which is consistent with experimental data and the wave functions are of proper symmetry. What is important, our approach requires neither additional computer time comparing to standard DFT calculations nor changes in the DFT codes. At the end we discuss the influence of spinorbit interaction on elastic properties of PbTe crystals.

\section{Calculations}

In calculations we used two $a b$ initio codes: OpenMx [4] and Quantum Espresso [5]. The first one uses atomic orbitals, the second one uses plane waves as the basis set for wave functions. The fully relativistic pseudopotentials are distributed only with OpenMx package. However, due to a very large number of valence electrons (20 for $\mathrm{Pb}$ and 16 for $\mathrm{Te}$ ), calculations for larger systems (mixed crystals) require a very large computational power. That is why, using programs distributed with the packages we generated fully relativistic pseudopotentials for $\mathrm{Pb}$ and $\mathrm{Te}$ with four and six valence electrons, respectively. In calculations we used gradient exchange-correlation functional of the form proposed by PerdewBurke-Ernzerhof [6] for Quantum Espresso and Local Density Approximation functional for OpenMX. The way we changed the strength of spin-orbit coupling was different for two codes. OpenMX directly offers possibility of controlling the spin-orbit strength for $l$-type orbitals by changing the splitting between semilocal parts of pseudopotentials for $j=l \pm 1 / 2$. The splitting is characterized by parameter $k_{\text {so }}$ with $k_{\text {so }}=1$ corresponding to the splitting obtained for an atom from solution of the Dirac equation. In calculations we were changing strength of spin-orbit coupling for lead $6 p$ levels because these levels are most important to the band structure calculations. 
In Quantum Espresso the way of fixing the strength of spin-orbit coupling is quite different. It is possible to change the value of speed of light constant $c_{\text {cau }}$ in the Dirac equation where $c_{\text {cau }} \approx 137$ corresponds to the true value of the speed of light in atomic units. In this way we change magnitude of all relativistic effects and in particular spin-orbit interaction strength.

\section{Results}

Figure 1 shows the dependence of the energy gap at $L$ point of the Brillouin zone on the strength of spinorbit interaction calculated using OpenMX and Quantum Espresso packages, respectively. We see that this dependence is qualitatively similar, although the energy gap does not disappear in the case of Quantum Espresso calculations. In the OpenMX package case it is possible to check the character of the wave functions for valence and conduction bands.
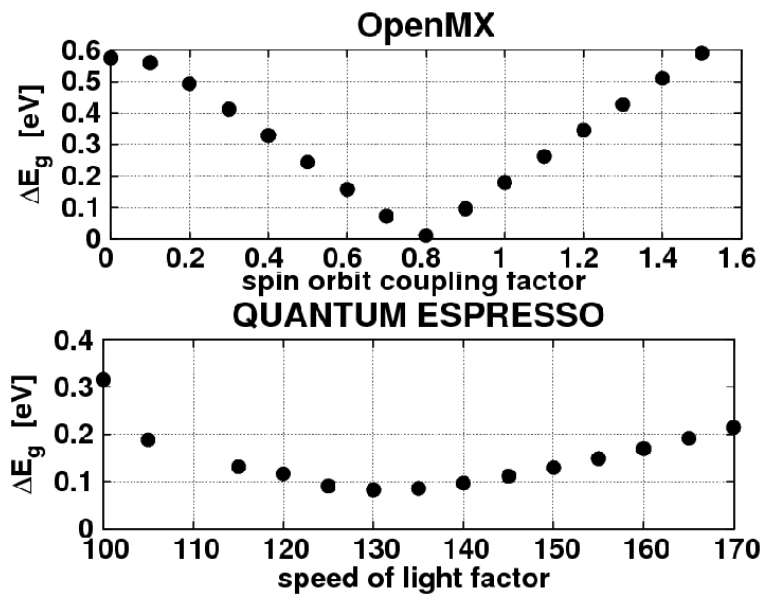

Fig. 1. Dependence of band gap energy $\Delta E_{\mathrm{g}}$ of strength on spin-orbit coupling strength in OpenMx and Quantum Espresso.

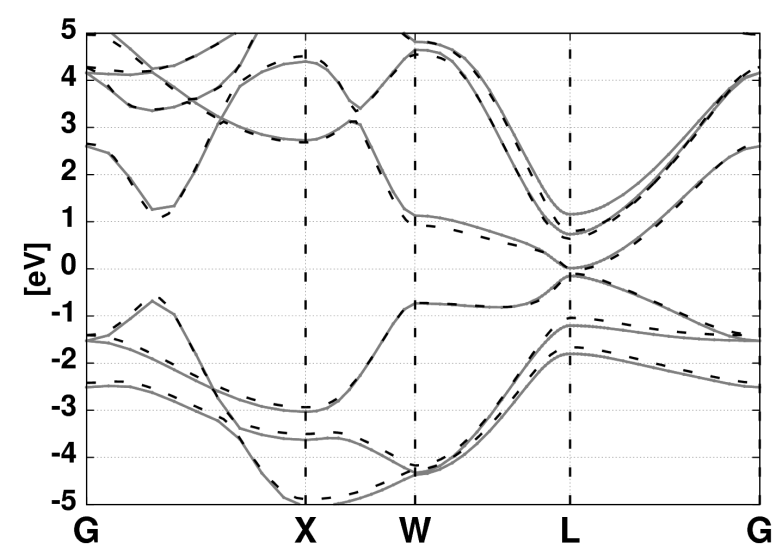

Fig. 2. Band structure of PbTe calculated with OpenMX (dashed line) and Quantum Espresso (solid line).

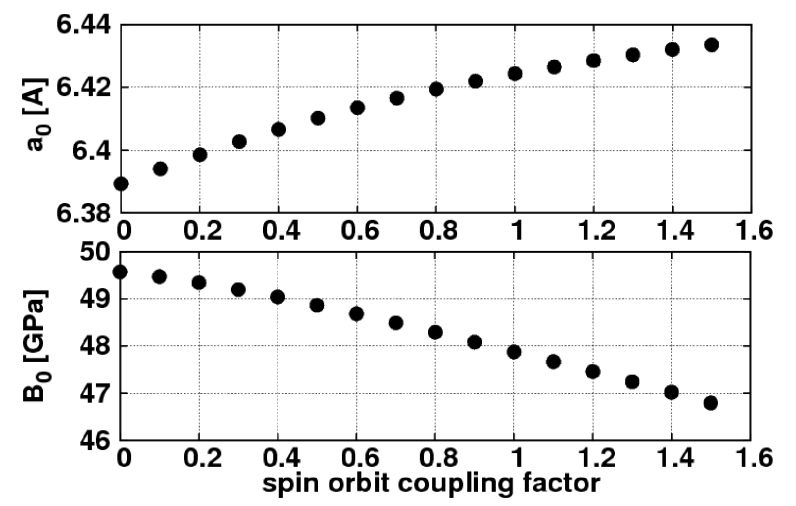

Fig. 3. Dependence of bulk modulus $B_{0}$ and equilibrium lattice constant $a_{0}$ on strength of spin-orbit coupling in OpenMx.

For $k_{\text {so }}<0.8$ the wave functions are of proper symmetry. In Fig. 2 we show that for $k_{\mathrm{so}}=0.6$ for OpenMX and for $c_{\text {cau }}=160$ for Quantum Espresso we obtain practically the same band structures along chosen directions of the Brillouin zone. The energy gap at the $L$ point $E_{\mathrm{g}} \approx 0.19 \mathrm{eV}$. Finally we checked the influence of spinorbit coupling strength on values of elastic parameters in PbTe: bulk modulus $B_{0}$ and equilibrium lattice constant $a_{0}$. This part of calculations was performed using OpenMX code. Experimental values of these parameters are: $B_{0}=41.6 \mathrm{GPa}$ and $a_{0}=6.46 \AA$. As it is shown in Fig. 3 for calculated range of $k_{\text {so }}$ parameter range of variation of $B_{0}$ was about $\approx 6.5 \%$ of experimental value. Similar analysis for $a_{0}$ dependence shows that range of variation of $a_{0}$ was about $\approx 0.7 \%$ of experimental value.

In the present work we showed that the problem of the inverted band structure may be overcome by a proper change of the spin-orbit coupling strength. We showed how to perform these changes in two ab initio packages and we discussed the influence of the spin-orbit coupling strength on the band structure and elastic properties of PbTe.

\section{Acknowledgments}

This material is based upon work supported by the research project No. 0992/T02/2007/32 of the Ministry of Science and Higher Education (Poland) and in part by the U.S. Army Research Laboratory and the U.S. Army Research Office under contract/grant number W911NF-08-1-0231.

\section{References}

[1] K. Hummer, A. Grüneis, G. Kresse, Phys. Rev. B 75, 195211 (2007).

[2] M. Städele, M. Moukara, J.A. Majewski, P. Vogl, Phys. Rev. B 59, 10031 (1999).

[3] S.-H. Wei, A. Zunger, Phys. Rev. B 55, 13605 (1997).

[4] www.openmx-sqare.org .

[5] www.pwscf.org .

[6] J.P. Perdew, K. Burke, M. Ernzerhof, Phys. Rev. Lett. 77, 3865 (1996). 